\title{
Rapid Prediction of Polyester Magnetic Tape Playability Using Water Contact Angles
}

\author{
ANDREW R. DAVIS, AES Associate Member \\ (adavi@loc.gov) \\ Preservation Research and Testing Division, Library of Congress, Washington, DC 20540-4560, USA
}

\begin{abstract}
The playability and degradation of polyester magnetic media has been an ongoing concern for audio curators, technicians, and hobbyists for decades. As these collections continue to age, users increasingly desire to transfer their contents. However, such a task can be daunting. The necessity of triaging collections for preservation is clear: institutions with large collections may not know the current condition of any given tape, and individual users may have limited equipment and remediation tools available at any given time. This report presents a new, rapid, non-technical tool for evaluating the playability and physical surface of polyester magnetic tapes without needing to place them on playback equipment or use expensive technical instrumentation. Water contact angle, using a small micro-liter sized droplet, was found to accurately predict the physical playback condition of the vast majority of tapes from a sampling of test tapes from the Library of Congress testing labs. This tool provides an appealingly simple and powerful method to directly probe a tape's physical surface. Results could frequently be interpreted by eye, without needing technical processing equipment or software. Total costs for testing equipment were found to be less than \$100 USD, allowing for access to a wide range of users.
\end{abstract}

\section{INTRODUCTION}

Audio users, collectors, and archival organizations have long known about the degradation of polyester based (or polyethylene terephthalate, PET, also commonly Mylar) magnetic tapes that incorporate polyester-urethane binder, historically used in many reel-to-reel audio tapes and audiovisual cassettes [1-4]. This degradation may render audio tapes entirely unplayable. But even when "playable" on a playback deck, degraded tapes can introduce noise into the original recordings, shed material from their surface, or lose their magnetic data coatings entirely. These problems can permanently ruin a tape and jeopardize its contents. A number of researchers have undertaken studies to understand the causes and characteristics of degraded polyester-based audio materials [4-11].

Despite previous and ongoing studies, uncertainties still remain in the exact details of tape degradation, especially on the scale of individual tapes. A single audio object's history, starting from slight differences in manufacturer formulation all the way to its up-to-date storage environment, is both unreasonable to assume and unlikely to be precisely known. Frustratingly, the state of degradation in a single specific tape may not be evident until that tape is on the playback deck. By Murphy's Law, this will invariably be the case for an institution's only copy of a unique recording. Furthermore, even if degradation is suspected, some tape users are hesitant to apply common remedial treatments unless absolutely necessary due to continued uncertainty about possible long-term consequences.

Given the inherent uncertainties of whether any individual tape will pose a playback problem, and given the caution from some audio users in using particular remedial treatments, a need seems to exist for predictive tools to assess tape degradation. Even more simply, not all organizations have the same breadth of resources to address degradation problems. Options for addressing tape degradation become more scarce and valuable when restricted by increasingly-hard-to-find playback equipment (which necessitates protection from sticky tape shed), limited personnel and expertise, and remediation methods that can be both time-intensive and equipment-intensive. Researchers have identified potential methods for identifying degraded tapes, but these methods presume a significant degree of technical know-how and possession of expensive analytical tools [6], [9-12]. A cheap, rapid, and non-technical tool to evaluate the condition of a tape prior to playback might tremendously aid technicians in their daily workflow and aid in the preservation of magnetic media.

The work presented here will describe how a droplet of water can be used as a simple tool to assess tape condition prior to playback and prior to treatment, for which no similarly simple predictive tool currently exists. Water contact angle is a tool used widely in the surface science fields 
to characterize the surface of a material, providing simultaneous insight into the chemical and physical properties of that surface [13-14]. This method involves application of a microliter-sized water droplet to the surface of interest, followed by evaluation of the droplet shape, either by qualitative visual inspection or by quantitative calculations. The shape of this droplet varies according to materials' surface properties and thus provides information about surface chemistry and texture. Since the vast majority of audio tape playback problems involve the interaction of a tape's surface with various playback heads, guides, and capstans, focused evaluation of that surface by contact angle seems especially appropriate.

\section{METHODS}

This study analyzed tapes available from the Center for the Library's Analytical Scientific Samples (CLASS), located in the Preservation Research and Testing Division at the Library of Congress [15]. The sample set ultimately included 53 test tapes, specifically chosen to represent different playability conditions, different manufacturers, and presence or absence of tape back-coat. All samples were 1/4" polyester-based tapes incorporating polyesterurethane binders, and sample tapes were assumed to match the boxes they were stored in. Throughout this report, "LC Tape ID\#" refers to the individual tape identification numbers within CLASS at the Library of Congress (LC).

On-deck tape playability condition was assessed for each tape using a modified Scully 280 tape deck, which included a tape path in contact with typical rollers, capstans, and guide posts, contacting both front and back surfaces of the tape. Tested tapes were played for a few minutes at both 7.5 IPS and 15 IPS, with occasional FF and RW. A tape was deemed unplayable if it exhibited any of the following conditions: (1) shedding or deposition of material from the tape onto playback equipment; (2) audible squealing; (3) slowdown of tape playback or winding. This evaluation of playback condition was purely physical and did not evaluate any audio content. Given ongoing efforts to define more accurate descriptors of tape degradation modes $[4,16]$, the term "unplayable" in this report will be used to encompass any or all of these problems.

For contact angle tests using a microliter-sized water droplet, the droplet was dispensed so it remained at the tip of the pipette dispenser. The suspended droplet was then gently touched to the surface of the tape, and the pipette tip was then removed leaving a droplet on the tape surface for analysis. Fig. 1a shows an example of a contact angle test in progress. Contact angle could then be quantified by calculating the angle formed between the surface and edge of the water droplet, as shown in Fig. $1 b$.

In this report, contact angle measurements were performed using a $5 \mu \mathrm{L}$ droplet of deionized water dispensed from a disposable glass micropipette with wire plunger. A few exploratory measurements were also made using conventional tap water, with no significant differences observed. Digital images were captured using a Carson MM640 Digital Microscope. Contact angle was then calculated

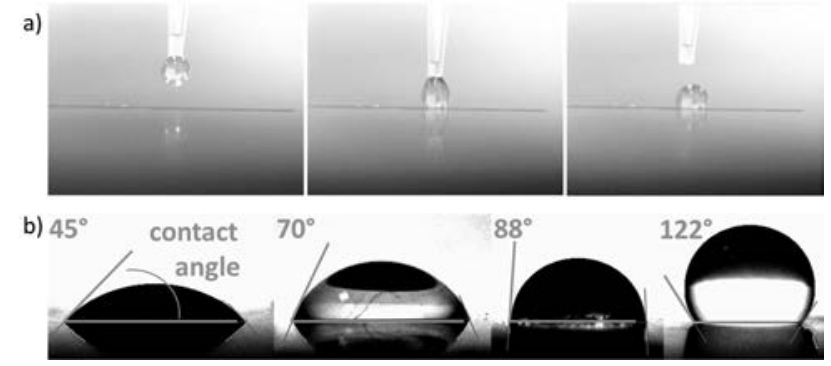

Fig. 1. Photographic images showing a) the process of contact angle drop dispensing, and b) an example of contact angle quantification.

by processing captured images with the open source program ImageJ and its "Contact Angle" plugin developed by Marco Brugnara [17]. However, in many cases, a "low" or "high" contact angle could be easily identified by eye alone. For each tape measured in this report, contact angle was measured five times, and the measurements were averaged. This was performed on both the oxide layer and the backside of each tape. All contact angles were measured within the first two meters of the tape start.

\section{RESULTS AND DISCUSSION}

\subsection{Initial Testing of Tape "Twins"}

Given variations in polyester tape manufacturers and formulations, we were particularly interested in playable/unplayable tape "twins" in our test collection, which were pairs of tapes of the same make and model where one tape demonstrated sticky or unplayable characteristics while the second tape did not. The contact angles measured for three of these tape twins are shown in Fig. 2. The results from this test showed that unplayable sticky tapes all had relatively low contact angles $\left(<90^{\circ}\right)$, while playable and non-degraded tapes had significantly higher contact angles. The two pairs of Ampex tapes are especially interesting, since their chemical and manufacturing formulation is likely very similar. Thus the difference between the two Ampex 406 tapes (and likewise the 456 tapes) is primarily due to playability and degradation factors.

A pair of 3M 227 tapes are shown here as a useful case study of the practical application of contact angle evaluation. One of the 3M 227 tapes (ID\# 1185) had not yet been checked for playability condition prior to measuring these contact angles, and its condition was unknown. The measurements in Fig. 2 were the first assessment of that tape, and these results strongly suggested that this tape was unplayable when compared to the other 3M 227 tape (as well as the other tape twins). Indeed, this tape left moderate amounts of oxide residues on the testing deck when played.

\subsection{Expanded Survey of Sample Tapes}

The initial results inspired confidence that contact angle measurements might be useful for general assessment of tape playability. However, the initial test cases 


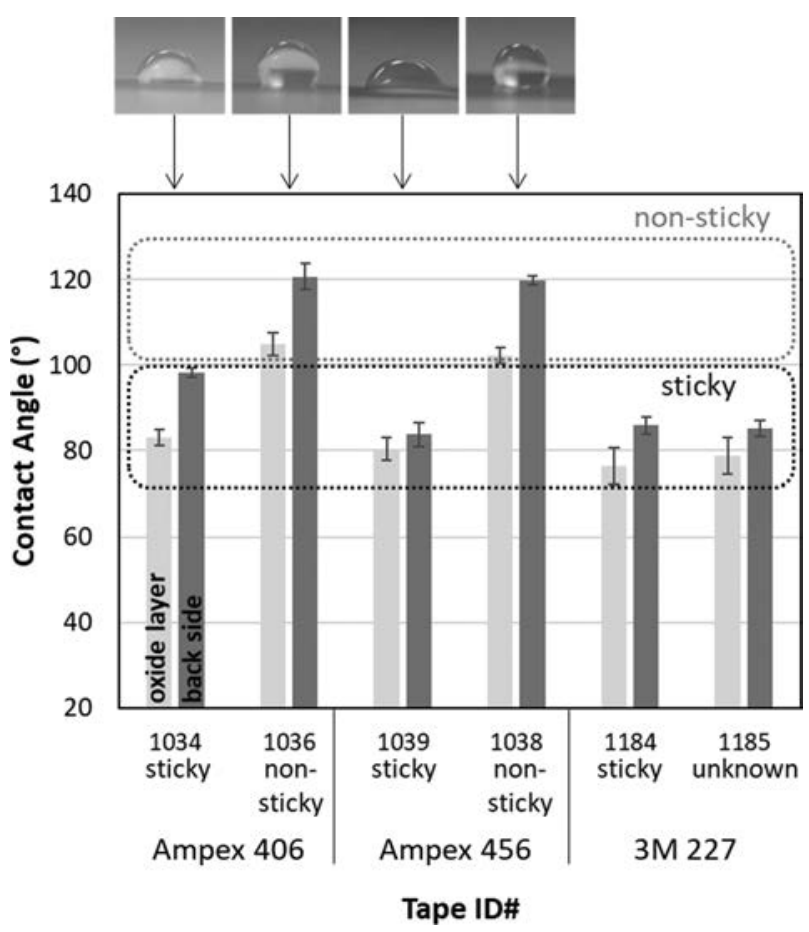

Fig. 2. Comparison of contact angle measurements on sticky and non-sticky "twin" tapes, with the visual appearance of contact angles shown in photographs above.

are a clearly limited sample set, constrained to carefully identified "twin" tapes of interest. To better evaluate the more general reliability of contact angle predictions in a practical setting, we expanded the test set to survey 53 tapes spanning different manufactures, presence (or lack) of back-coat, and manufacturing time periods.
As before, these tapes were tested for playability, and contact angles were measured on both sides of each. Some of these tapes had previously been tested for playability and some had not. Contact angles from this larger selection of tapes is shown in Fig. 3, with physically unplayable tapes noted by a solid bold outline.

As seen in Fig. 3, a simple contact angle measurement was found to accurately correlate and predict tape playability for every single test sample of $3 \mathrm{M}$ and Ampex tapes. For these tapes, contact angles lower than $100^{\circ}$ reliably indicated an unplayable tape. By contrast, cleanly playable tapes showed contact angles significantly larger than $100^{\circ}$. Most of these differences in contact angle were easily observable by eye and did not need quantification for successful evaluation. 3M tapes tended to have a closer gap between the contact angles of playable and unplayable tapes, but the relationship still held true. This trend was also generally true for Quantegy tapes, however some Quantegy tapes showed similar contact angles yet different playability condition. These similar contact angles were all less than $100^{\circ}$, and it might be that the "playable" tapes with these relatively low contact angles are approaching an unplayable condition. That said, the majority of tested Quantegy tapes showed a similar and unambiguous connection between contact angle and playability.

BASF brand tapes were the one significant outlier in these test results. While contact angle was able to accurately and reliably predict the playability condition of $3 \mathrm{M}$, Ampex, and Quantegy tapes, it was more difficult to assess the playability of BASF. This difficulty was partly due to the fact the BASF tapes in the sample set did not show any unambiguously "sticky" or unplayable behavior. These tapes also all showed contact angles close to $100^{\circ}$. So contact angles may be predictive, but without reliably unplayable

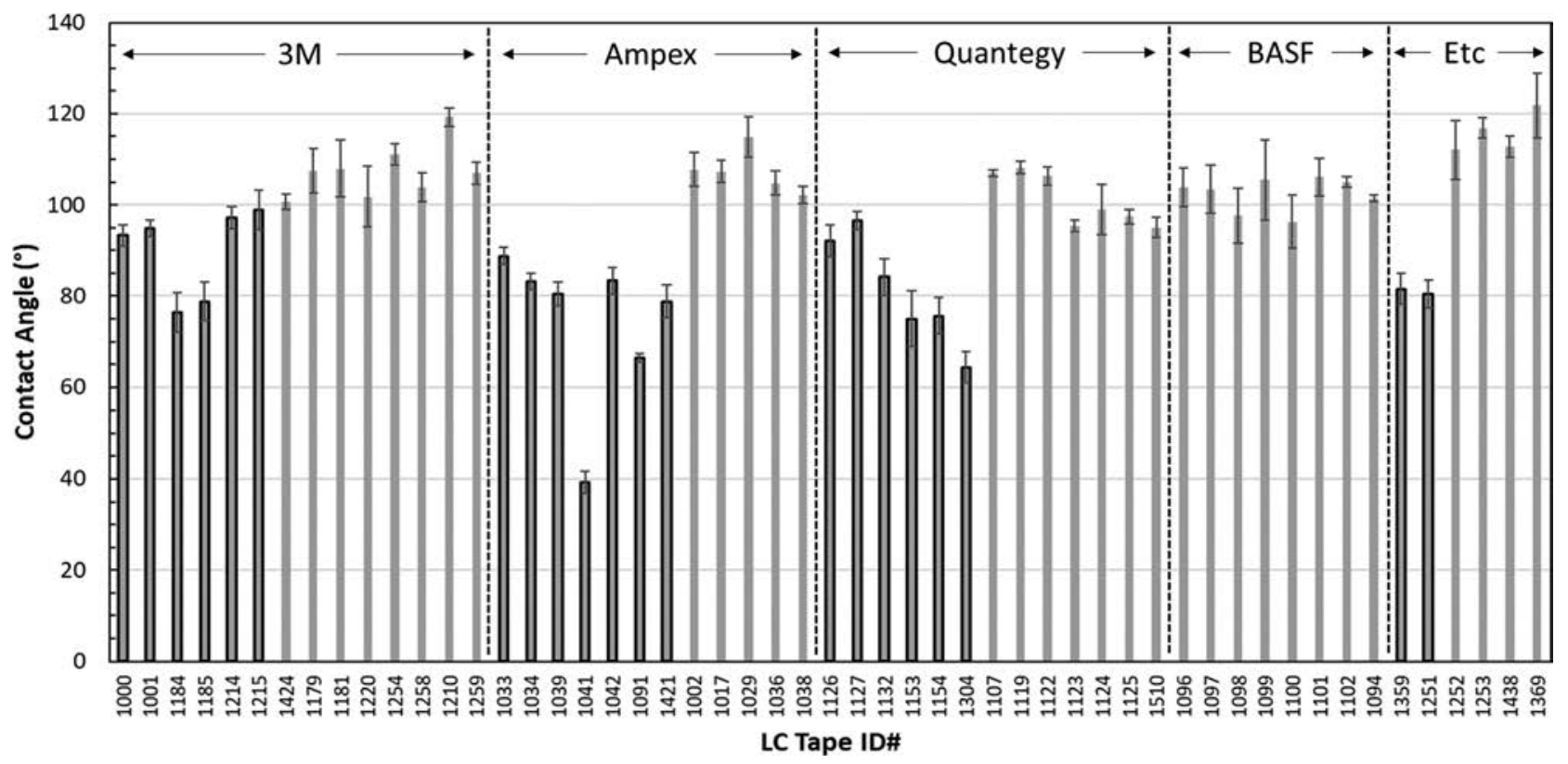

Fig. 3. Contact angles measured on the oxide layer for an expanded survey of test tapes, sorted by manufacturer. Bold outlines indicate unplayable tapes. "Etc." includes tapes from Maxell, Sony, and RadioShack. 
Table 1. Summary of average contact angle for all unplayable and playable tapes of each manufacturer, with number of correct contact angle predictions of playability condition

\begin{tabular}{|c|c|c|c|}
\hline \multirow[b]{2}{*}{ Tape brand } & \multicolumn{2}{|c|}{ Avg. contact angle $\left(^{\circ}\right)$} & \multirow{2}{*}{$\begin{array}{c}\% \text { correct } \\
\text { predictions (out } \\
\text { of total samples) }\end{array}$} \\
\hline & unplayable & playable & \\
\hline $3 \mathrm{M}$ & $90( \pm 10)$ & $107( \pm 6)$ & $100 \%(14 / 14)$ \\
\hline Ampex & $74( \pm 17)$ & $107( \pm 5)$ & $100 \%(12 / 12)$ \\
\hline Quantegy & $81( \pm 12)$ & $102( \pm 6)$ & $77 \%(10 / 3)$ \\
\hline BASF & n.a. & $103( \pm 3)$ & $75 \%(6 / 8)$ \\
\hline Other & 91 & $116( \pm 4)$ & $100 \%(6 / 6)$ \\
\hline ALL & $81( \pm 13)$ & $106( \pm 7)$ & $90 \%(47 / 53)$ \\
\hline
\end{tabular}

tapes, this was difficult to evaluate for certain. These observations also raise interesting questions about what might be different in the BASF tapes to cause this different behavior, and additional work might clarify this observation.

It is interesting to consider the subtleties of this data set when acknowledging that tapes degrade gradually and in different ways. The data in Fig. 3 are only a snapshot at the current time, and these tests may have captured some tapes that are nearly, but not yet, unplayable. For example, two Quantegy tapes, LC ID\#s 1127 and 1123 (Quantegy 456 and 406 , respectively), showed nearly identical contact angles. However when tested for playback, tape ID\#1127 was deemed unplayable while tape ID\#1123 was found to be cleanly playable. The fact that both of these tapes had a contact angle between $90^{\circ}$ and $100^{\circ}$, very close to the range where tapes were observed to begin to become unplayable, poses the question of whether tape ID\#1123 might soon show playback problems too. If so, these analyses might prove additionally useful in helping to triage tapes for transfer and preservation.

Table 1 summarizes the average contact angles for the measured test tapes. Contact angle was able to accurately predict the playability condition of all $3 \mathrm{M}$ and Ampex tapes tested. This table makes clear the wide variation in contact angle for sticky or unplayable tapes. This variation is visually evident from the tape-by-tape data shown in Fig. 3 , where unplayable tapes frequently skew downward to very low contact angles. By contrast, cleanly playable tapes tend to cluster narrowly around contact angles above $100^{\circ}$. These variations might be a promising indicator that certain types of tape degradation affect contact angles differently, and that these measurements might provide additional use in learning and differentiating between degradation modes (and whether specific remedial treatments would be effective).

At this point, an anecdotal observation is noteworthy. While testing the 53 tapes of this survey, we initially observed a "failure" rate greater than $15 \%$ for using contact angle as a predictor for tape condition. That is, assuming a low contact angle indicated an unplayable tape, contact angle measurements failed to predict the actual playability condition more than $15 \%$ of the time. However, careful re-inspection of the "erroneous" tape frequently revealed the tape had its condition mislabeled, was returned to the wrong box, or had simply become unplayable in the intervening years since its initial labeling. After observing these behaviors and identifying older errors in bookkeeping, the predictive "failure" rate from contact angle testing dropped to $10 \%$. If the sample BASF tapes are removed from consideration (due to their anomalous contact angle results), contact angle was able to accurately predict the playability of $93 \%$ of test tapes (and 100\% of Ampex and $3 \mathrm{M}$ tapes). When considered from a viewpoint of practical use, it struck us as a perfect and powerful example that contact angle measurements were able to indicate many tapes whose condition had changed or had been mislabeled.

\subsection{Effects of Backcoating}

Fig. 4 shows a reorganization of the data from Fig. 3, sorted by tapes with or without backcoats. The contact angles measured on the oxide surface showed no discernible correlation or trend with the presence or absence of a backcoat. As a brief aside: the tested tapes' playability show that we encountered some unplayable tapes without any backcoat. This is a clear indicator that users should be cautious before subscribing to blanket assumptions that backcoats are a primary cause of tape degradation, which was an assumption we occasionally encountered. Clearly, tapes without backcoats can become unplayable.

Contact angles were also measured on the back side of each sample tape, with results shown in Fig. 5 (similarly grouped by tapes with or without backcoats).

No correlation was found between back side contact angle and tape playability. Instead, a different trend was found from the back sides of backcoated and non-backcoated tapes. For tapes without backcoats, contact angles on the tapes' back side showed very little variation from tape to tape regardless of manufacturer or playable condition. However, back side contact angles varied widely for all backcoated tapes. These contact angles on backcoated tapes generally mirrored the contact angles seen on the oxide surface of the same tapes. The consistency in contact angle on the backs of non-backcoated tapes can likely be explained by the fairly hydrophilic PET base film that is known to remain fairly stable compared to other tape components [18]. The variation in contact angle observed on the back side of backcoated tapes is a more curious phenomenon. We can hypothesize a number of causes: residual material from the oxide layer may be contaminating the surface of the backcoat; the backcoat itself might be degrading; the tape-to-tape and manufacturer-to-manufacturer variations in backcoat texture may be affecting water droplet wettability $[13,14,19]$. Results from this study alone cannot yet offer a definitive explanation for the variations seen on backcoated tapes. Regardless, these variations suggest that contact angle measurements might be able to identify more complicated interactions of the backcoat with other surfaces.

\subsection{Dynamic Contact Angles}

The contact angle analyses presented so far have all been static contact angles. That is, contact angles were measured 


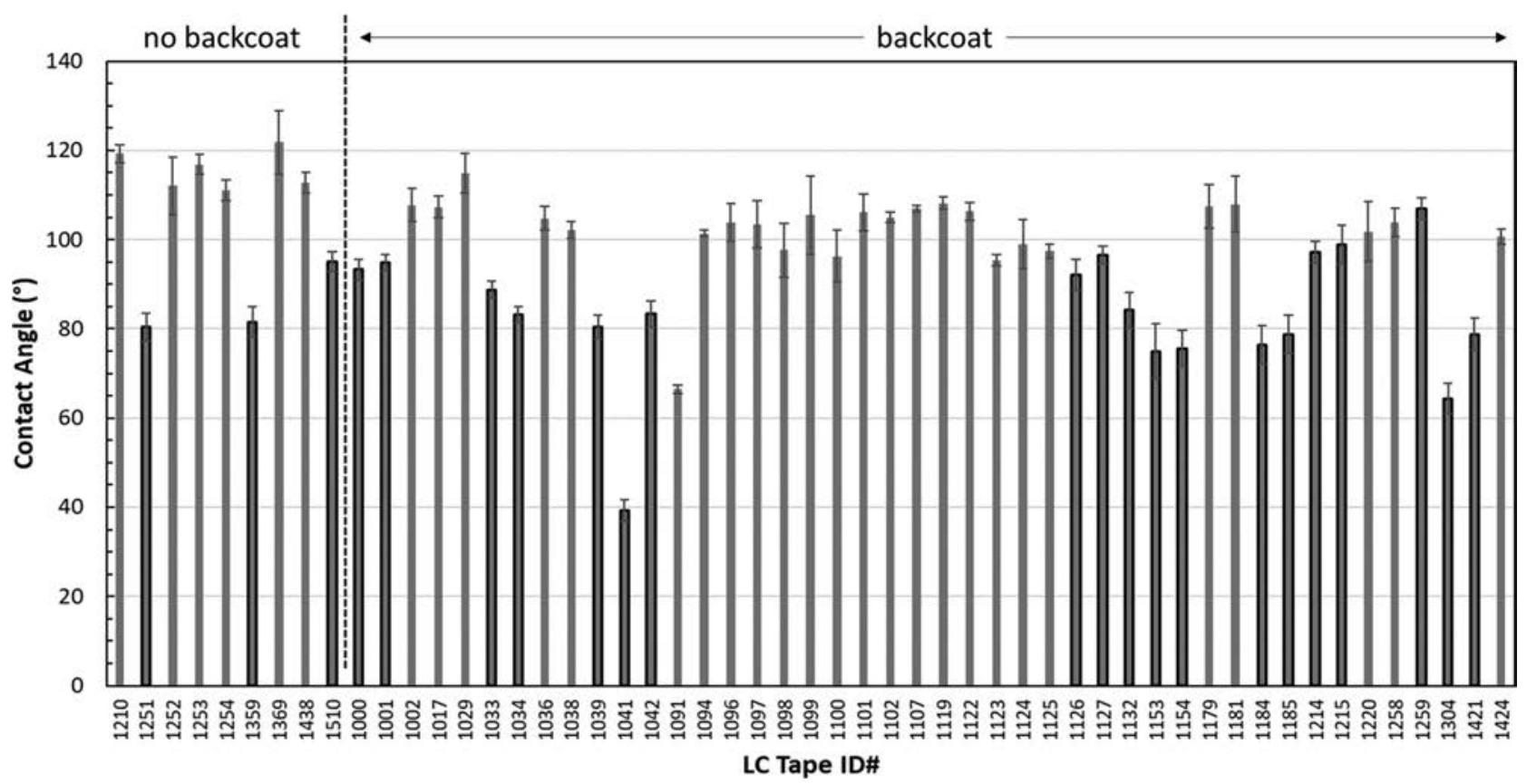

Fig. 4. Contact angles on front (oxide) side of tapes with and without backcoats. Bold outlines indicate unplayable tapes.

at a single moment in time, shortly after dispensing the water droplet. However, we noticed that in many unplayable or sticky tapes, the water droplets continued to wet out across the tape surface and decreased in contact angle over time. This was rarely observed to occur in well-behaved playable tapes. This phenomenon is shown in Fig. 6, where two tapes showed similar contact angles immediately after dispensing a droplet. In this case the playable Quantegy 407 tape retained approximately the same contact angle shape, while the shedding Quantegy 456 tape showed a contact angle which decreased over the course of one minute. Contact angles for these and two additional tapes are plotted as a function of time in Fig. 7. The contact angles in the unplayable tapes rapidly decreased in the first 20 seconds after dispensing the test droplet.

All "static" contact angles reported in Figs. 3-5 were measured after 30 seconds of equilibration time. Based on the data in Fig. 7, this was likely sufficient to account for the largest changes in contact angle over time. Additional work examining the time-based dynamics of contact angle and surface wetting on degraded tapes might provide additional insight into different modes of degradation (e.g., onset of binder hydrolysis, contamination from back coatings, or surface defects from general wear).

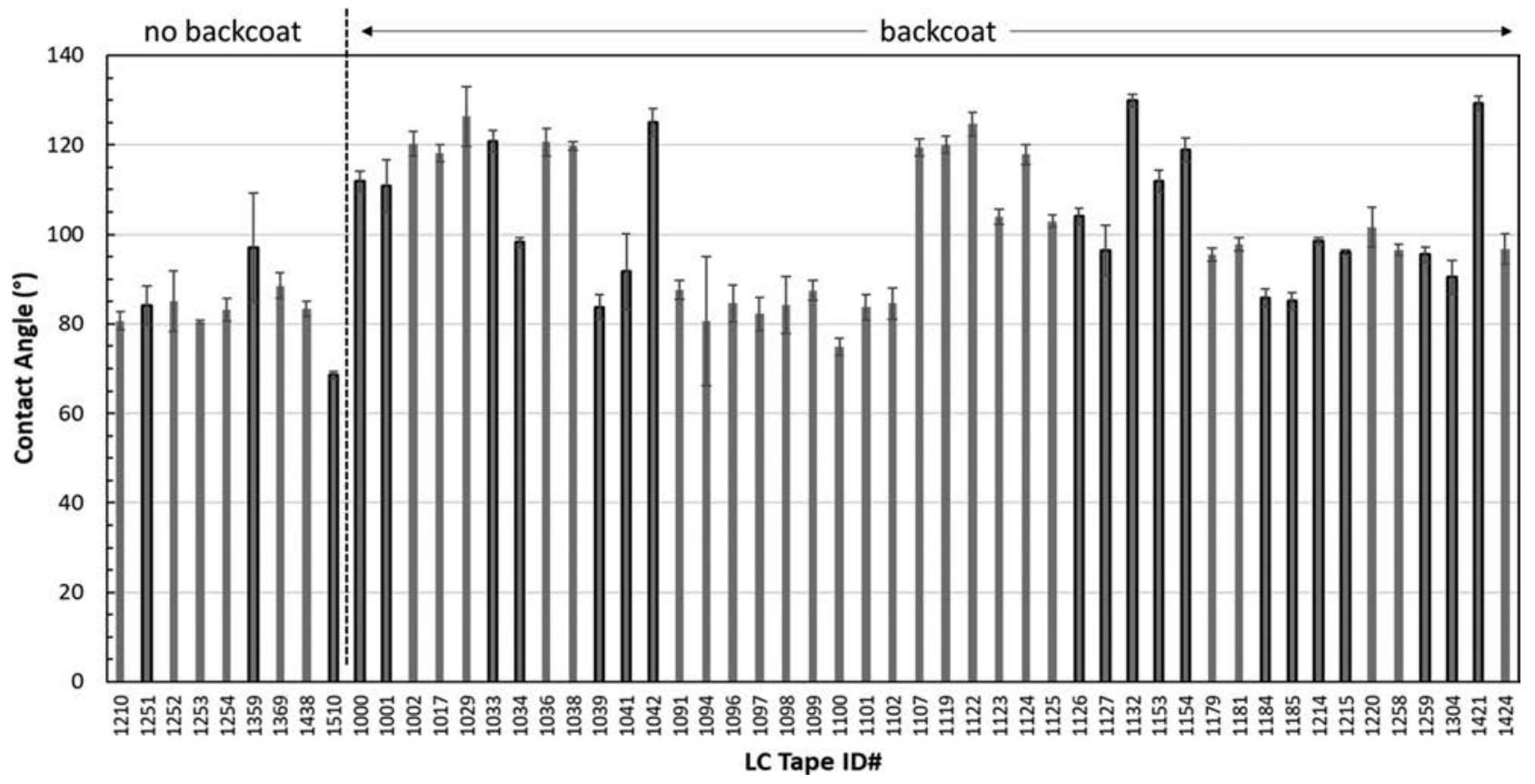

Fig. 5. Contact angles on back side of tapes with and without backcoats. Bold/thick outlines indicate unplayable tapes. 

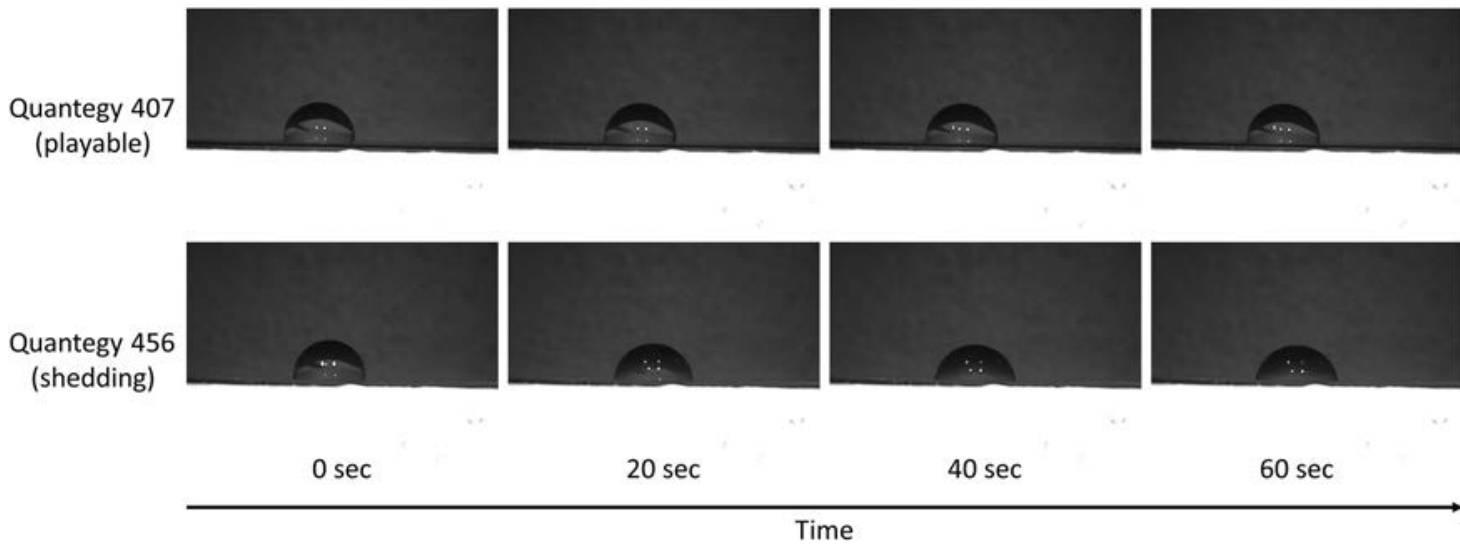

$40 \mathrm{sec}$

$60 \mathrm{sec}$

Time

Fig. 6. Response of contact angle droplet shape over 60 seconds for a playable tape and an unplayable shedding tape.

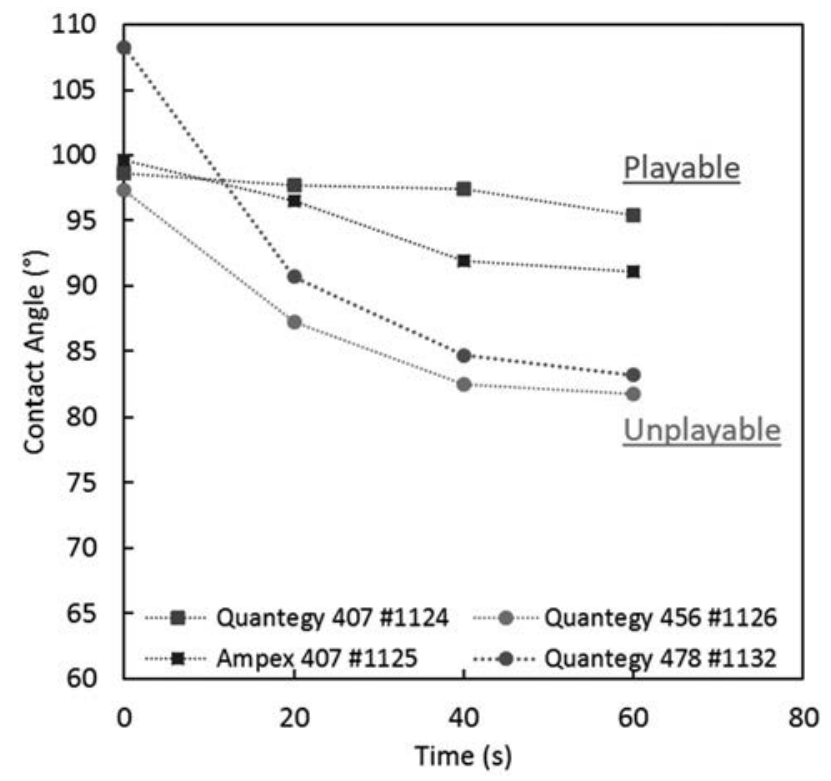

Fig. 7. Contact angle as a function of time for two cleanly playable tapes (square symbols) and two shedding and sticky tapes (circle symbols).

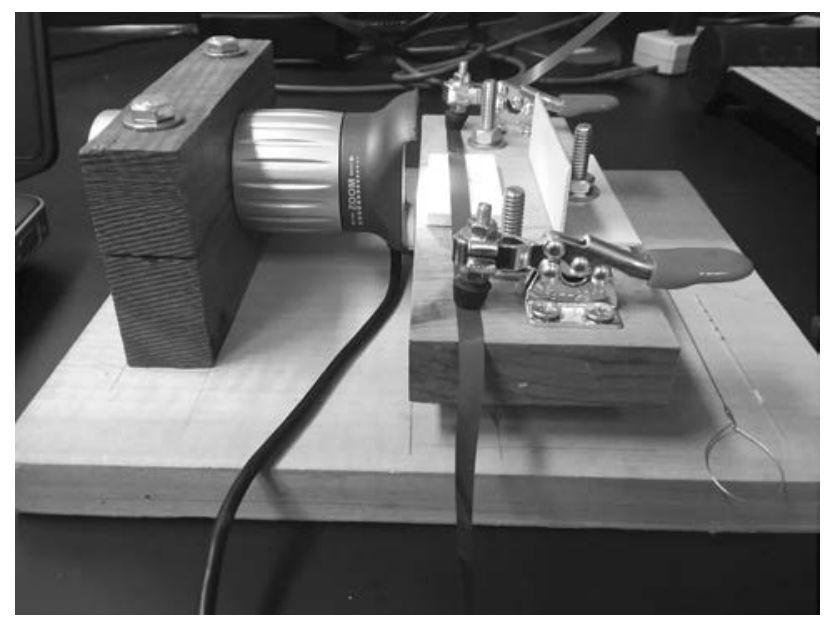

Fig. 8. Low cost $(<\$ 100)$ contact angle test rig prototyped by Mike Rivers.

\section{LOW-COST TESTING OPTIONS}

Finally, since this work was inspired by a desire to expand test options for audio users with limited resources and equipment, we wanted to explore options for an easily attainable testing system. A simple low-cost proof-of-concept tool was prototyped by Mike Rivers and shown in Fig. 8 . This design shows how high-cost tools are not necessary, using a USB microscope camera $(<\$ 60), 5 \mu \mathrm{L}$ glass capillary micropette and wire plunger $(<\$ 20$ for set of 100$)$, and small toggle clamps for holding the sample tape flat $(<\$ 20)$.

\section{CONCLUSIONS}

As magnetic tape users can appreciate: tapes are idiosyncratic. They each have their own histories, may be part of a different production lot, or may not match the label on their box. For this reason it cannot always be assumed that a tape of a "known" problematic model is indeed unplayable when considering an individual tape in one's collection. This is especially concerning for audio users with limited availability of resources, who might need to be especially concerned about best use of their available personnel or equipment, or who may not have access to high throughput facilities.

The results of this study showed that contact angle measurements could be a useful tool for a rapid, simple, lowcost evaluation of a polyester-based tape's condition. In general, a low contact angle was found to correspond to degraded and difficult-to-play tapes, while high contact angles corresponded to cleanly playable tapes. These differences were frequently discernible by eye alone. The tests had some degree of dependence on the tape's manufacturer, but overall trends remained highly predictive for most of the tapes tested. Subtleties in the data suggest that, with further work, we may be able to use contact angle data for evaluating specific types of degradation or for the triage of prioritizing tapes to transfer and preserve.

The method presented here for using contact angles as a predictive tool may not perfectly translate from these test tapes to an individual user's unique collection. However, given uncontrolled collection-to-collection variables, the 
results presented here have strong robust trends which we anticipate will be useful for many audio tape users.

Finally, the surface-focused approach of this analytical tool is especially compelling and attractive. The physicality of tapes and playback equipment necessitates surface contact, and these surfaces are ultimately the most common sites of material failure. A better understanding of tapes' surfaces will undoubtedly lead to improved preservation of audio-visual heritage content.

\section{ACKNOWLEDGMENTS}

Considerable thanks must be given to Mike Rivers for his enthusiasm and initiative in building his own demonstration tool. Additional thanks to Richard Hess, Peter Alyea, Rob Friedrich, and Brad McCoy for insightful discussions and suggestions. And a final thank you to Prof. Tom McCarthy, whose jesting comments at an ACS meeting provided the initial inspiration for this work.

\section{REFERENCES}

[1] D. Brown, R. Lowry, and L. Smith, "Prediction of the Long Term Stability of Polyester-Based Recording Media.pdf," Natl. Bureau of Standards, NBSIR 84-2988 (1984 Dec.).

[2] B. Bhushan, Tribology and Mechanics of Magnetic Stoarge Devices, 2nd ed. (New York: Springer-Verlag, 1996).

[3] B. Bhushan, Mechanics and Reliability of Flexible Magnetic Media, 1st ed. (New York: Springer-Verlag, 2000).

[4] R. L. Hess, "Tape Degradation Factors and Challenges in Predicting Tape Life," ARSC J., vol. 39, no. 2, pp. 240-275 (2003).

[5] A. R. Davis, E. Monroe, and F. G. France, "Understanding Magnetic Tape Degradation by Polymeric and Material Testing," presented at the 2018 AES International Conference on Audio Archiving, Preservation \& Restoration (2018 Jun.), conference paper P-1.

[6] S. Hobaica, "Analysis of Audio Magnetic Tapes with Sticky Shed Syndrome by ATR-FTIR," J. Appl. Polym. Sci., vol. 128, no. 3, pp. 1962-1973 (2013 Aug.). https://doi.org/10.1002/app.38364

[7] E. Cuddihy, "Aging of Magnetic Recording Tape," IEEE Trans. Magn., vol. 16, no. 4, pp. 558-568 (1980 Jul.). https://doi.org/10.1109/TMAG.1980.1060652
[8] D. Schüller, "Magnetic Tape Stability: Talking to Experts of Former Tape Manufacturers," IASA J., vol. 42, pp. 32-36 (2014 Jan.).

[9] F. Bressan, R. Bertani, C. Furlan, F. Simionato, and S. Canazza, "An ATR-FTIR and ESEM Study on Magnetic Tapes for the Assessment of the Degradation of Historical Audio Recordings," J. Cult. Herit., vol. 18, pp. 313-320 (2016 Mar.) https://doi.org/10.1016/j.culher.2015.09.004

[10] E. Gómez-Sánchez et al., â€œATR/FT-IR Spectroscopy for the Characterisation of Magnetic Tape Materials," Spectrosc. Eur., vol. 24, no. 1, p. 6-12 (2012 Feb.).

[11] F. Bressan, R. L. Hess, P. Sgarbossa, R. Bertani. "Chemistry for Audio Heritage Preservation: A Review of Analytical Techniques for Audio Magnetic Tapes," Heritage, vol. 2, pp. 1551-1587 (2019 May). https://doi.org/10.3390/heritage2020097

[12] B. M. Cassidy et al., â€œMinimally Invasive Identification of Degraded Polyester-Urethane Magnetic Tape Using Attenuated Total Reflection Fourier Transform Infrared Spectroscopy and Multivariate Statistics," Anal. Chem., vol. 87, no. 18, pp. 9265-9272 (2015 Sep.) https://doi.org/10.1021/acs.analchem.5b01810

[13] P.-G. de Gennes, F. Brochard-Wyart, and D. Quere, Capillarity and Wetting Phenomena: Drops, Bubbles, Pearls, Waves (New York: Springer-Verlag, 2004).

[14] M. E. Schrader and G. I. Loeb, Eds., Modern Approaches to Wettability: Theory and Applications (New York: Plenum Press, 1992).

[15] "Center for the Library's Analytical Scientific Samples (CLASS)," Library of Congress. Online. Accessed 18 March 2019. http://www.loc.gov/preservation/ scientists/projects/class.html.

[16] F. Bressan, "A Framework for the Description of Age-Related Symptoms in Audio Media: Definition and Implementation," Studies in Conserv. (2019 Jul.). https://doi.org/10.1080/00393630.2019.1641666

[17] M. Brugnara, Contact Angle. 2006. Image J. Online. Accessed 18 March 2019. https://imagej.nih.gov/ ij/plugins/contact-angle.html

[18] M. Edge, M. Mohammadian, M. Hayes, N. Allen, K. Brems, and K. Jones, "Aspects of Polyester Degradation: Motion Picture Film and Videotape Materials," J. Imaging Sci. Technol., vol. 36, no. 1, pp. 13-20 (1992).

[19] R. N. Wenzel, "Surface Roughness and Contact Angle," J. Phys. Chem., vol. 53, no. 9, pp. 1466-1467 (1949). https://doi.org/10.1021/j150474a015 


\section{THE AUTHOR}

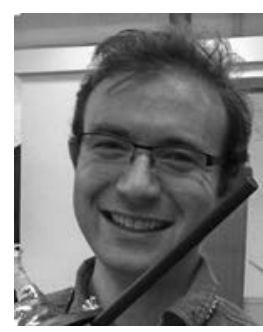

Andrew R. Davis

Dr. Andrew R. Davis is a chemist and polymer scientist in Library of Congress's Preservation Research and Testing Division. He is currently focused on collections preservation by studying the fundamental degradation science of polymer-based materials, including paper, film, and modern media. His work has included research and presentations on magnetic audio tapes, repair adhesives, and the test books from the William Barrow laboratory. Andrew is also involved in work to better understand the role of light, oxygen, and the environment in the fugitive nature of materials in order to better enable public display of light-sensitive objects. Andrew received his Ph.D. in polymer science and engineering at the University of Massachusetts Amherst. Prior to the Library of Congress, he has worked in the Central Research Laboratories of 3M where he researched how photochemical processes affect polymers, adhesives, and colored materials. Andrew is actively involved in STEM outreach programs and laboratory volunteer opportunities for high school and college students. 